\title{
Optical Excitation of a Forbidden Magnetic Resonance Mode in a Doped Lutetium-Iron-Garnet Film via the Inverse Faraday Effect
}

\author{
A. H. M. Reid, ${ }^{1,2, *}$ A. V. Kimel, ${ }^{1}$ A. Kirilyuk, ${ }^{1}$ J. F. Gregg, ${ }^{2}$ and Th. Rasing ${ }^{1}$ \\ ${ }^{1}$ Radboud University Nijmegen, Institute for Molecules and Materials, Heijendaalseweg 135, 6525 AJ Nijmegen, Netherlands \\ ${ }^{2}$ Department of Condensed Matter Physics, Clarendon Laboratory, University of Oxford, \\ Parks Road, Oxford OX13PU, United Kingdom \\ (Received 11 February 2010; revised manuscript received 2 July 2010; published 3 September 2010)
}

\begin{abstract}
The effective magnetic field induced by a femtosecond pulse of circularly polarized light, via the inverse Faraday effect, is shown to excite a magnetic-dipole forbidden exchange spin resonance in a lutetium iron garnet. An external magnetic field cannot excite this mode, as the iron sublattices have the same gyromagnetic ratio and no net torque can be applied between them. However, since the sublattices have different magneto-optical susceptibilities, the inverse Faraday effect induces different effective fields on different iron sites, allowing excitation.
\end{abstract}

PACS numbers: 78.47.J-, 76.70.Hb, 78.20.Ls

A recent exciting development in solid-state physics has been the discovery of ultrafast paths for the control of magnetism by light. This area has developed rapidly, from the first discovery of ultrafast demagnetization in nickel [1], through the demonstration of methods to coherently excite magnetic resonances [2,3], to the demonstration of alloptical magnetic switching in $\mathrm{GdFeCo}$ by a 40 fs circularly polarized optical pulse $[4,5]$. These latter experiments have demonstrated the ability of light to act as an effective magnetic field on a femtosecond time scale via the inverse Faraday effect (IFE). It should be stressed that this effect does not originate in the light's magnetic field, but is instead due to a nonlinear interaction with the electric field. It thus remains an open question whether or not the description of the IFE as an effective magnetic field is always sufficient. In this Letter we demonstrate that the physics of the IFE is richer than simply generating an effective magnetic field, as we observe the excitation of a magneticdipole forbidden exchange resonance in lutetium iron garnet by a circularly polarized optical pulse. The results show that the IFE leads to the generation of two site-specific effective magnetic fields by the same optical pulse.

The ultrafast IFE has been demonstrated in a number of materials, such as: $\mathrm{DyFeO}_{3}[2],(\mathrm{LuYBi})_{3}[\mathrm{FeGa}]_{5} \mathrm{O}_{12}$ [6], $\mathrm{FeBO}_{3}$ [7] and $\mathrm{GdFeCo}$ [8]. Microscopically, the effect can be described as either optical Stark splitting of spin sublevels or as Raman scattering on magnons, depending of whether the optical field is applied parallel or perpendicular to the magnetic quantization axis. Phenomenologically, it is described in terms of the action of an effective magnetic field pulse present for the duration of the optical field.

The system used in this study is a doped film of lutetium iron garnet (LuIG) grown on a GGG substrate. The film is $7 \mu \mathrm{m}$ thick and has the composition $\left[\mathrm{Lu}_{1.69} \mathrm{Y}_{0.65} \mathrm{Bi}_{0.66}\right]$ $\left(\mathrm{Fe}_{3.85} \mathrm{Ga}_{1.15}\right) \mathrm{O}_{12}$. The $\mathrm{Lu}, \mathrm{Y}$, and $\mathrm{Bi}$ ions are surrounded by eight oxygen ions in a dodecahedron-this is the garnet's (c) site [see Fig. 1(a)]. As neither Lu, Y nor Bi possess a magnetic moment, they do not directly contribute to the magnetization. The magnetic $\mathrm{Fe}$ ions are distributed between two sites: a tetrahedral-coordinated oxygen " $d$ " site and an octahedral-coordinated " $a$ " site. These moments are antiferromagnetically coupled, causing them to anti align, while ferromagnetic coupling between sites of the same symmetry forms two ferromagnetic sublattices. A prevalence of tetrahedral sites, three for every two octahedral sites, causes the overall order to be ferrimagnetic $\left(T_{C}=400 \mathrm{~K}\right)$. Nonmagnetic $\mathrm{Ga}$ ions substitute onto $\mathrm{Fe}$ sites, diluting the two magnetic sublattices. At the concentration used, Ga shows an $88 \%-90 \%$ preference for the

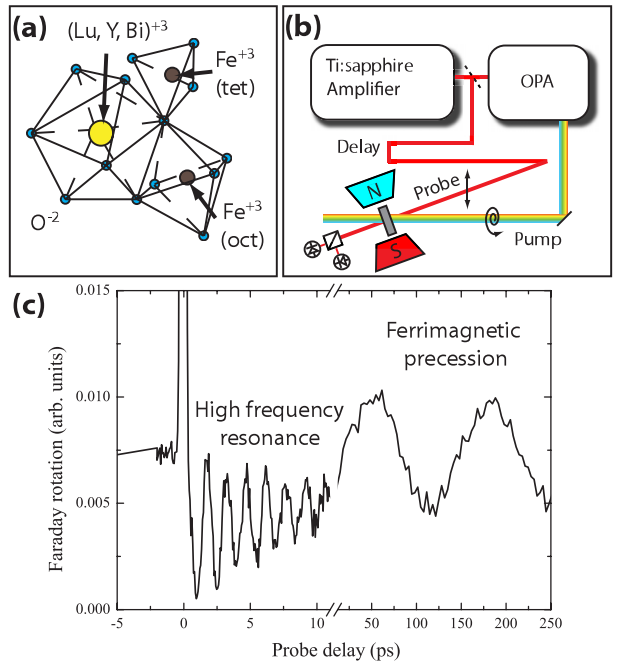

FIG. 1 (color online). (a) The crystal structure of the garnet. (b) A schematic diagram of the time-resolved pump-probe measurement preformed on the LuIG. (c) A time-resolved measurement of the Faraday rotation of LuIG following a right-hand circularly polarized pump pulse at $80 \mathrm{~K}$. Two distinct modes are observed; the ferrimagnetic resonance mode triggered by the IFE (frequency $=7.21 \mathrm{GHz}$ at $\mathbf{B}=0.3 \mathrm{~T}$ ) and a previously unobserved high-frequency mode (frequency $=671 \pm 3 \mathrm{GHz}$ ). 
tetrahedral site and thus reduces the garnet's saturation magnetization [9].

The optical properties of iron garnets have been well documented [10,11]. It is known that the Faraday effect stems from differences in the transitions for right and left circularly polarized light on the Fe sites [10]. The difference in symmetry between these sites manifests as a difference in the crystal-field splitting of excited states; thus the absorption spectra also differ. For the same reason, the contribution to the Faraday rotation from octahedral and tetrahedral $\mathrm{Fe}$ is not equal. In pure $\mathrm{Y}_{3} \mathrm{Fe}_{5} \mathrm{O}_{12}$ (YIG) the Faraday rotation from octahedral sites is about 1.9 times as strong as that from tetrahedral sites [12]. It is for this reason that the Faraday effect is not a reliable indicator of the garnet's magnetization [13]. This does pose an intriguing question: will the IFE also act differently on different $\mathrm{Fe}$ sites, unlike an external magnetic field?

To address this question, time-resolved pump-probe measurements are made of the response of the LuIG sample to a pulse of circularly polarized light; see Fig. 1(b). The sample is mounted in a liquid-nitrogen cooled cryostat. An external field is applied in the plane of the sample to orientate the magnetization. A linearly polarized probe pulse is directed to pass through the garnet film at normal incidence; this allows the measurement of the out of plane component of the magnetization via the Faraday effect. The probe pulse wavelength is centered at $800 \mathrm{~nm}$ with a bandwidth of $30 \mathrm{~nm}$ and a duration of $60 \mathrm{fs}$. An optical parametric amplifier is used to vary the pump wavelengths in the range $530-750 \mathrm{~nm}$. The pump pulse, which generates the IFE, passes through the sample making an angle of about ten degrees with the surface normal. Both pump and probe pulses are adjusted to spatially overlap within the film with a diameter of approximately $100 \mu \mathrm{m}$.

In an applied magnetic field, precession at the ferrimagnetic resonance frequency appears after excitation with circularly polarized light; see Fig. 1(c). However, in the same measurements another resonance mode is observed at a frequency of $0.7 \mathrm{THz}$ in the first few picoseconds following the pump pulse. This high-frequency resonance bears several striking similarities to the induced ferrimagnetic resonance. First, it shows a $180^{\circ}$ phase shift between excitations with right and left circularly polarized light, see Fig. 2(a), as is observed with the ferrimagnetic mode [3]. Second, it disappears, like the (IFE induced) ferrimagnetic resonance, for linearly polarized pump light. In addition, its amplitude is linearly dependent on the pump fluence without displaying any threshold behavior. The frequency of this new mode is also observed to be independent of the spectral variation of the pump pulse (Fig. 2 inset). No resonant spectral behavior is observed which excludes the possibility of it being due to the beating of excited states [14]. In addition, no variations are seen in its amplitude or frequency for changes in the external field up to $0.3 \mathrm{~T}$.

The frequency of this new mode $(650 \mathrm{GHz}$ at $300 \mathrm{~K})$ does not correspond to any of the garnet's optical phonon
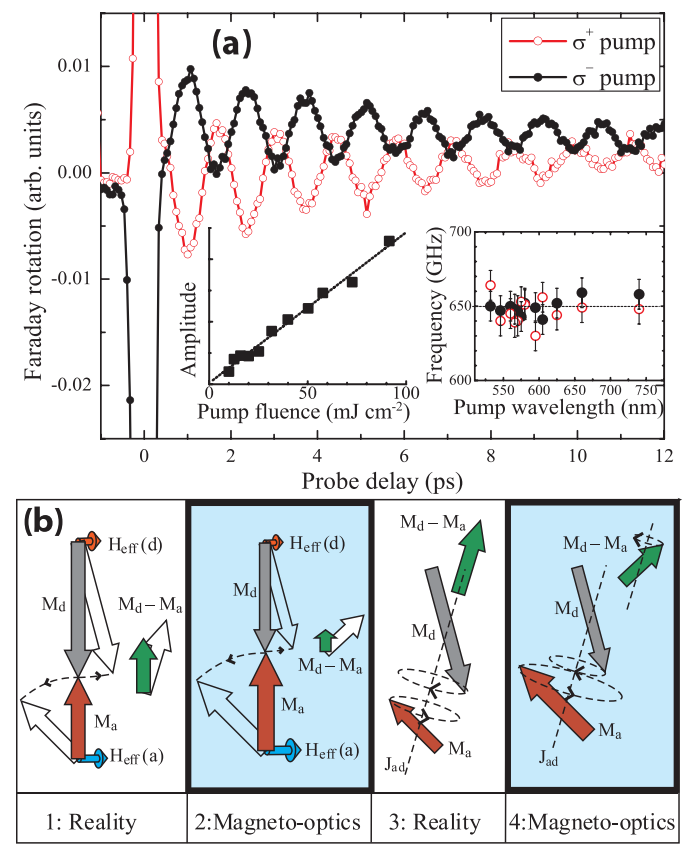

FIG. 2 (color online). In (a) the high-frequency resonance mode is shown for right and left circularly polarized pump light at a temperature of $140 \mathrm{~K}$. The inset graphs show the mode's amplitude dependence on the pump fluence and the spectral dependence of its frequency at $300 \mathrm{~K}$. In (b), a cartoon illustrates the nature of the exchange resonance and how it is excited. Panel 1 shows how different effective fields acting on the tetrahedral $\left(M_{d}\right)$ and octahedral $\left(M_{a}\right)$ sites can induce a canting between the sublattices, and in panel 3, how these moments precess around this exchange field $\left(J_{a d}\right)$. The blue/gray panels 2 and 4 illustrate how the reality of panels 1 and 2 are distorted in a magnetooptical experiment-the $M_{a}$ moments appear enhanced with respect to $M_{d}$. Note that this distortion leads to an apparent precession of the resultant moment around the exchange field.

modes (these typically appear above $3 \mathrm{THz}$ [15]), and is also above the frequency of the optically accessible acoustic phonons. The latter would also be expected to show a strong wavelength dispersion which is not observed; see Fig. 2(a) inset. The optical branches of the magnon spectrum can be similarly excluded as a possible explanation. The lowest of these appears at a frequency corresponding to $\simeq 10 J_{a d}\left(J_{a d}\right.$ being the interlattice exchange interaction) [16]; this is about $6 \mathrm{THz}$ in Fe garnets [17]. In fact only one excitation is expected in this spectral range, a KaplanKittel $(K-K)$ exchange resonance between the tetrahedral and octahedral Fe sublattices.

The $K-K$ exchange resonance is a magnetic excitation which occurs between two coupled magnetic sublattices in ferrimagnets with a frequency proportional to the exchange coupling constant [18]. The origin of this mode can be related to a canting of the two sublattices away from their mutual exchange interaction. Both sublattices then experience a torque due to the other's exchange field. A solution of the coupled torque equations shows that both magnetic sublattices precess around the average direction of the 
exchange field, which is parallel to the ferrimagnetic moment, illustrated in Fig. 2(b). The frequency of this precession is given by

$$
\omega_{\mathrm{ex}}=\gamma \lambda_{a d}\left|\mathbf{M}_{d}-\mathbf{M}_{a}\right|,
$$

where $\gamma$ is the gyromagnetic ratio of iron, $\lambda_{a d}$ is the molecular field (exchange) constant and $\mathbf{M}_{d}, \mathbf{M}_{a}$ are the sublattice magnetizations. Using a value of $\lambda_{a d}=920$ from Dionne et al. [19] for $90 \% \mathrm{Ga}$ doping, and the garnet's measured saturation magnetization of $\mu_{0} \mathbf{M}=$ $0.027 \pm 0.003 \mathrm{~T}$, yields: $\omega_{\text {ex }} / 2 \pi=0.70 \pm 0.08 \mathrm{THz}$.

Exchange resonances are well known in $\mathrm{Fe}$ garnets containing an additional magnetic sublattice of rare-earth ions. In that case, the difference in gyromagnetic ratios between the rare-earth and the iron ions allows the exchange resonance to be magnetically excited; however, without this difference, no torque can be exerted by a magnetic field and the mode is forbidden. Therefore, the exchange mode is not normally observed between iron sublattices. However, this does not apply to the case of excitation by the IFE. Here the different magneto-optical susceptibilities of the sublattices gives rise to different local effective fields, generated by the same optical pulse.

Solving the equations of motion for magnetic sublattices subject to different effective fields shows that they precess at the frequency given by Eq. (1). It should be stressed here, however, that the overall magnetization does not precess as it remains oriented along the exchange direction; see Fig. 2(b). This is consistent: a resonance that cannot be excited by a time-varying magnetic field cannot generate a rotating magnetic moment that would emit magnetic radiation at the same frequency. However, the mode can still be observed; this is again because each sublattice has a different magneto-optical susceptibility, and, therefore, the moment on the octahedral site appears enhanced with respect to that on the tetrahedral site. Figure 2(b) illustrates how the overall magnetic moment thus appears to be rotating around the exchange field because of the difference in magneto-optical susceptibilities.

As the $K$ - $K$ resonance mode has not (to our knowledge) been observed between the iron sublattices of a garnet, there is no experimental way to check our hypothesis. Instead, the best that can be achieved is a comparison with theory. The equation for the resonance frequency is [18]

$$
\begin{aligned}
f_{\text {ex }}= & \frac{g \mu_{B} J_{a d}}{2 \pi \hbar} \mid \mathbf{M}_{d}\left(J_{a d}, J_{d d}, T, \% \mathrm{Ga}\right) \\
& -\mathbf{M}_{a}\left(J_{a d}, J_{a a}, T, \% \mathrm{Ga}\right) \mid,
\end{aligned}
$$

where $J_{a d}, J_{a a}$, and $J_{d d}$ are the intersite exchange interactions, and the magnetization is given by [19]

$$
\begin{aligned}
\mathbf{M}_{x}\left(J_{a d}, J_{x x}, T, \% \mathrm{Ga}\right)= & \mathbf{M}_{x 0}(\% \mathrm{Ga}) B_{J}\left(\frac { g \mu _ { B } S } { k _ { B } T } \left(J_{a d} M_{y}\right.\right. \\
& \left.\left.+J_{x x} M_{x}\right)\right),
\end{aligned}
$$

where $x=a, d ; x \neq y=a, d ; S$ is the spin quantum num- ber, and $B_{J}$ is the Brillouin function. It is easy to see that Eq. (2) has a highly nonlinear dependence on the exchange interactions, as these also serve to determine the sublattice magnetizations. The magnitude of the exchange interactions is not directly accessible and can only be extracted by fitting the temperature dependencies of the saturation magnetization and spin-wave spectrum [20]. Comparing our experimental results with theory is therefore not easy. However, using the best data available such a comparison is attempted. An empirical expression for the variation of YIG's exchange parameters with Ga doping was determined by Dionne [19]. Here the presence of $\mathrm{Bi}$ in the measured garnet is not taken into account, but is expected to cause a slight increase in the exchange parameters in comparison to those observed in Ga doped YIG [21]. The exact distribution of $\mathrm{Ga}$ is also unknown, but the tetrahedral site preference is expected to be nearly $90 \%$ at the garnet's doping level [9]. It is observed that even a $1 \%$ change in the Ga preference between sites causes a $20 \%$ change in the exchange resonance frequency. Figure 3(a) shows a comparison between the measured temperature dependence and the frequency calculated from Eq. (2). As expected, Eq. (2) cannot exactly predict the frequencies observed in the experiment though the overall correspondence is good. A tetrahedral Ga substitution between $88 \%$ and $89 \%$ gives the closest match to the experimental frequencies. However, the shape of the temperature dependence of the frequency is more consistent with that observed at about $90 \%$. This apparent under estimation of the

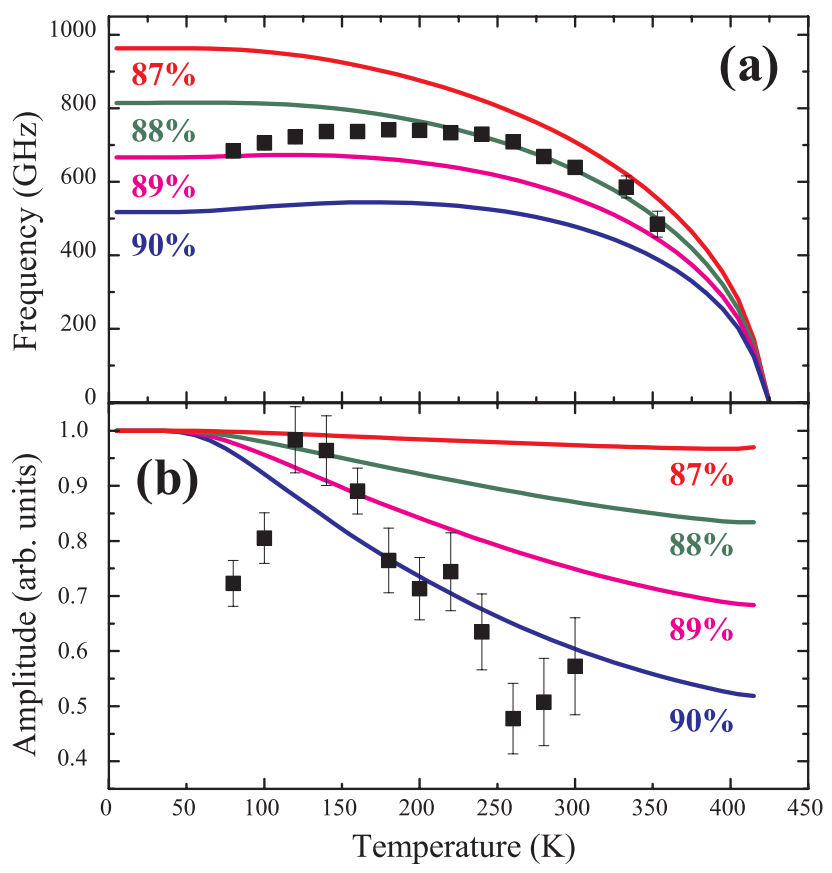

FIG. 3 (color online). (a) A comparison between the measured resonance frequency and that predicted by Eq. (1) for various proportions of $\mathrm{Ga}$ on the tetrahedral site. (b) The predicted amplitude is compared to measurements for the same concentrations. 


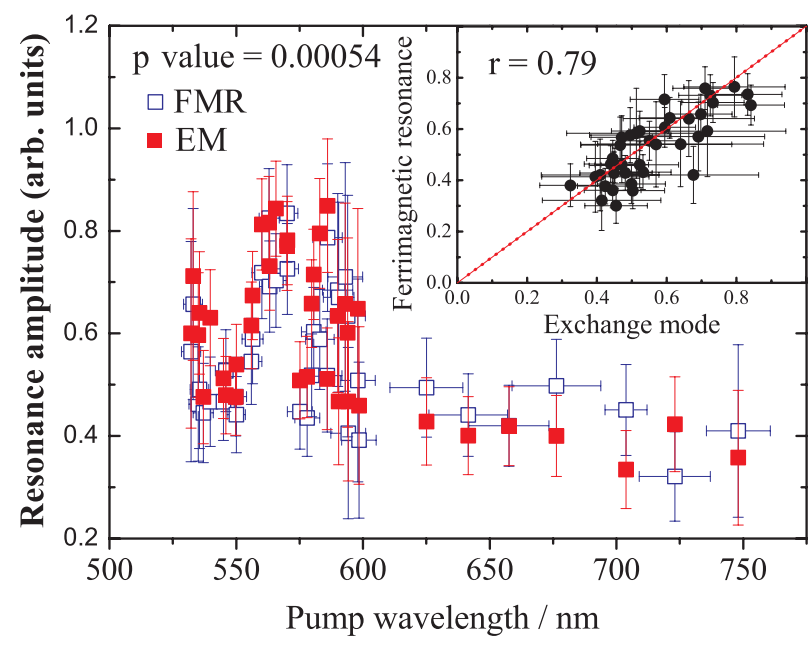

FIG. 4 (color online). The amplitude of the IFE induced ferrimagnetic resonance mode (FMR, open squares) and the exchange mode (EM, filled squares) is shown over the spectral range $530-750 \mathrm{~nm}$. The inset graph shows the correlation between the amplitudes.

exchange frequency is consistent with $\mathrm{Bi}$ enhancing the exchange coupling which is not accounted for in the model.

The amplitude of the resonance is also examined as a function of temperature; see Fig. 3(b). This is determined by fitting a damped sinusoid to the measured data and extrapolating its amplitude at zero delay. The damping is observed to increase strongly with increasing temperature and is also a significant source of error in the measured amplitude. This data is compared to the amplitudes extracted from numerical solutions of the two coupled torque equations which describe the sublattice magnetization dynamics. These equations were solved for the case of excitation by different effective field pulses on each sublattice. For simplicity, it is assumed that the effective fields acting on each sublattice are not dependent on temperature or sublattice magnetization. Figure 3(a) shows a comparison between measurement and simulation. The closest agreement between measurements and the model is again observed for $90 \% \mathrm{Ga}$ doping. The disagreement between the measurements and the model could be an indication that the effect of temperature on the magneto-optical susceptibility cannot be neglected.

To further test if the exchange resonance is really related to the IFE, the spectral correlation between the amplitudes of the exchange and ferrimagnetic resonances are examined in Fig. 4. A hypothesis test of the correlation coefficient $(r=0.79)$ gives the probability of correlation to within $0.5 \%$.

In this Letter we have demonstrated that femtosecond pulses of circular polarized light can excite a magneticdipole forbidden exchange resonance between the spins of two Fe sublattices via the inverse Faraday effect (IFE). This is allowed because the IFE is generated locally, as it depends on the magneto-optical susceptibility of each magnetic site. In LuIG this leads to tetrahedral and octahedral $\mathrm{Fe}$ sites experiencing inequivalent optically induced effective magnetic fields, resulting in a canting between the magnetic sublattices. The precession of these canted moments in their mutual exchange field corresponds to the exchange resonance mode, which, to the best of our knowledge, has not been previously observed. The very precise measurement of this resonance frequency gives a direct, and highly accurate, measure of the exchange interaction between the Fe sublattices. This demonstrates a versatility of femtosecond opto-magnetism beyond that of standard magnetic fields.

The authors thank J.H. Mentink and G. A. Brooker for discussions, A. Toonen and A. van Etteger for technical support, and J. Kisielewski for magnetometry data. This work was supported by the European networks UltraMagnetron (Grant NMP3-SL-2008-214469), FANTOMAS (No. 214810), and DYNAMICS, and the Dutch national organizations for scientific research FOM and NWO.

\footnotetext{
*a.reid@science.ru.nl
}

[1] E. Beaurepaire, J.-C. Merle, A. Daunois, and J.-Y. Bigot, Phys. Rev. Lett. 76, 4250 (1996).

[2] A. V. Kimel et al., Nature (London) 435, 655 (2005).

[3] F. Hansteen, A. Kimel, A. Kirilyuk, and T. Rasing, Phys. Rev. B 73, 014421 (2006).

[4] C. D. Stanciu et al., Phys. Rev. Lett. 99, 047601 (2007).

[5] K. Vahaplar et al., Phys. Rev. Lett. 103, 117201 (2009).

[6] F. Hansteen, A. Kimel, A. Kirilyuk, and T. Rasing, Phys. Rev. Lett. 95, 047402 (2005).

[7] A. M. Kalashnikova et al., Phys. Rev. Lett. 99, 167205 (2007).

[8] C. D. Stanciu et al., Phys. Rev. Lett. 98, 207401 (2007).

[9] S. Geller, J. A. Cape, G. P. Espinosa, and D. H. Leslie, Phys. Rev. 148, 522 (1966).

[10] A. K. Zvezdin and V.A. Kotov, Modern Magnetooptics and Magneto-optical Materials (Institute of Physics Publishing, Bristol and Philadelphia, 1997), Chap. 9, p. 163.

[11] G. B. Scott, D. E. Lacklison, and J. L. Page, Phys. Rev. B 10, 971 (1974).

[12] W. A. Crossley, R. W. Cooper, J. P. Page, and R. P. van Stapele, Phys. Rev. 181, 896 (1969).

[13] H. Matthews, S. Singh, and R. C. LeCraw, Appl. Phys. Lett. 7, 165 (1965).

[14] J. H. Versluis et al., Phys. Rev. B 80, 235326 (2009).

[15] J.-J. Song et al., J. Opt. Soc. Am. 63, 1135 (1973).

[16] W. Brinkman and R. J. Elliott, J. Appl. Phys. 37, 1457 (1966).

[17] V. Cherepanov, I. Kolokolov, and V. L'vov, Phys. Rep. 229, 81 (1993).

[18] J. Kaplan and C. Kittel, J. Chem. Phys. 21, 760 (1953).

[19] G. F. Dionne, J. Appl. Phys. 41, 4874 (1970).

[20] R. Gonano, E. Hunt, and H. Meyer, Phys. Rev. 156, 521 (1967).

[21] P. Novák, J. Englich, and H. Lütgemeier, Phys. Rev. B 37, 9712 (1988). 